\title{
Performance of window synchronisation in coherent optical ofdm system
}

\author{
Sofien Mhatli ${ }^{1}$, Bechir Nsiri ${ }^{2}$ \\ ${ }^{1}$ SERCOM, EPT \\ Université de Carthage, 2078, La Marsa, Tunis , Tunisia \\ ${ }^{2}$ Sys'com Lab, ENIT, BP.37 Le Belvédère 1002 \\ Tunis, Tunisia
}

\author{
Mutasam Jarajreh ${ }^{3}$, Basma Hammami $^{2}$, Rabah Attia $^{1}$ \\ ${ }^{3}$ Faculty of Engineering \& Environment, Northumbria \\ University, Ellison Place, Newcastle upon Tyne, NE1 \\ 8ST, UK
}

presented and discussed in Section 4. Finally, Section 5 concludes the work.

\section{COFDM SYSTEM DESCRIPTION}

Figure 1 shows the conceptual diagram of a generic COOFDM system, including five basic functional blocks: RF OFDM transmitter, RF-to-optical (RTO) up-converter, optical link, optical-to-RF (OTR) down-converter, and RF OFDM receiver. In the RF OFDM transmitter, the input digital data are first converted from serial to parallel into a "block" of bits consisting of Nsc information symbol, each of which may comprise multiple bits for m-ary coding. This information symbol is mapped into a two-dimensional complex signal Cki, for instance, using Gray coding, where Cki stands for the mapped complex information symbol. The subscripts of Cki correspond to the sequence of the subcarriers and OFDM blocks. The time domain OFDM signal is obtained through inverse discrete Fourier transform (IDFT) of Cki , and a guard interval is inserted to avoid channel dispersion [6,7]. The resultant baseband time domain signal can be described as :

$$
s_{B}(t)=\sum_{i=-\infty}^{+\infty} \sum_{k=-\frac{N_{S C}}{2}+1}^{k=N_{s c} / 2} c_{k i} \Pi(t-T s) e^{j 2 \pi f_{k}\left(t-i T_{s}\right)}
$$

Where:

$$
\begin{array}{r}
f_{k}=\frac{k-1}{t_{s}} \\
\Pi(t)=\left\{\begin{array}{l}
1,\left(-\Delta G<t \leq t_{s}\right. \\
0, t \leq-\Delta G, t>t_{s}
\end{array}\right.
\end{array}
$$

where Cki is the $\mathrm{i}^{\text {th }}$ information symbol at the $\mathrm{k}^{\text {th }}$ subcarrier; $f_{k}$ is the frequency of the $\mathrm{k}^{\text {th }}$ subcarrier; is the number of OFDM subcarriers Ts, $\Delta \mathrm{G}$, and ts are the OFDM symbol period, guard interval length, and observation period, respectively; and $\Pi(t)$ is the rectangular pulse waveform of the OFDM symbol. The extension of the waveform in the time frame of $[-\Delta \mathrm{G}, 0]$ in (1) represents the insertion of the cyclic prefix, or guard interval. The digital signal is then converted to an analog form through a DAC and filtered with a low-pass filter to remove the alias signal. The baseband OFDM signal can be further converted to an RF pass band through an RF IQ. The subsequent RTO up-converter transforms the base band signal to the optical domain using an optical IQ modulator comprising a pair of Mach-Zehnder modulators (MZMs) with 
a 90 degree phase offset. The baseband OFDM signal is directly up-converted to the optical domain given by:

$$
E(t)=e^{j\left(\omega_{L D 1} t+\varphi_{L D 1}\right)} \cdot s_{B}(t)
$$

Where $\omega_{L D 1}$ and $\varphi_{L D 1}$, respectively, are the angular frequency and phase of the transmitter laser. The up-converted signal $\mathrm{E}(\mathrm{t})$ traverses the optical medium with an impulse response of $\mathrm{E}(\mathrm{t})$, and the received optical signal becomes:

$$
E^{\prime}(t)=e^{j\left(\omega_{L D 1} t+\varphi_{L D 1}\right)} \cdot s_{B}(t) \otimes h(t)
$$

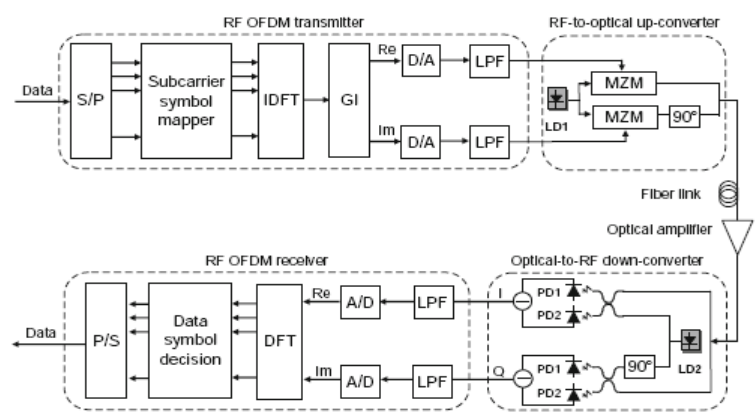

S/P: Serial-to-parallel GI: Guard time insertion (I)DFT: (inverse) Discrete Fourier transform D/A: Digitalto-analog LPF: Low pass filter. MZM: Mach-Zehnder modulator PD: Photodiode
LD: Laser diode

Fig. 1 Conceptual diagram for a generic CO-OFDM system with a direct up/down conversion architecture

Where $\otimes$ stands for convolution. The optical OFDM signal is then fed into the OTR downconverter, where the optical OFDM signal is converted to an RF OFDM signal. The directly down-converted signal can be expressed as:

$$
\begin{gathered}
r(t)=e^{j\left(\omega_{o f f} t+\Delta \varphi\right)} \cdot r_{0}(t) \\
r_{0}(t)=s_{B}(t) \otimes h(t) \\
\omega_{o f f}=\omega_{L D 1}-\omega_{L D 2} \\
\Delta \varphi=\varphi_{L D 1}-\varphi_{L D 2} \\
c_{k i}^{\prime}=e^{j \Phi_{i}} e^{j \Phi_{D}\left(f_{k}\right)} T_{k} c_{k i}+n_{i} \\
\Phi_{D}\left(f_{k}\right)=\pi \cdot c \cdot D_{t} \cdot \frac{f_{j}^{2}}{f_{0}^{2}} \\
c_{k i}^{\prime}=E^{\prime}(t)
\end{gathered}
$$

Where $\omega_{\text {off }}$ and $\Delta \varphi$ are respectively the angular frequency offset and phase offset between the transmitted and receive lasers. $\Phi_{D}\left(f_{k}\right)$ Is the phase dispersion due to the fiber chromatic dispersion [8]. $\Phi_{i}$ Is the ofdm common phase error (CPE) [9] due to the phase noises from lasers and RF local oscillators at both the transmitter and receiver.

\section{COFDM SYSTEM SYNCHRONISATION DESCRIPTION}

In the RF OFDM receiver, the down-converted OFDM signal is first sampled with an ADC. Then the signal needs to go through the following three levels of sophisticated synchronizations before the symbol decision can be made:

- DFT window synchronization in which OFDM symbols are properly delineated to avoid intersymbol interference

- Frequency synchronization, namely frequency offset $\omega_{\text {off }}$ being estimated, compensated, and, preferably, adjusted to a small value at the start.

- The subcarrier recovery, where each subcarrier channel is estimated and compensated.

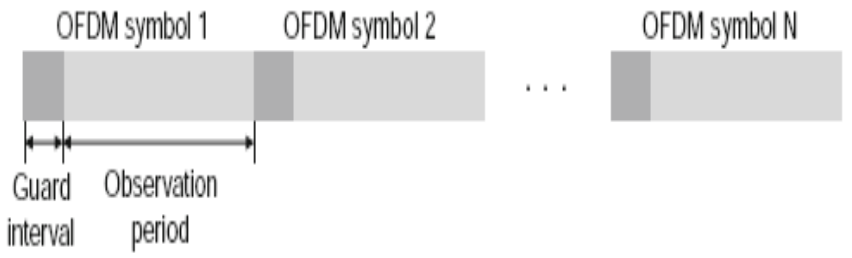

Fig. 2 Time domain structure of an OFDM signal

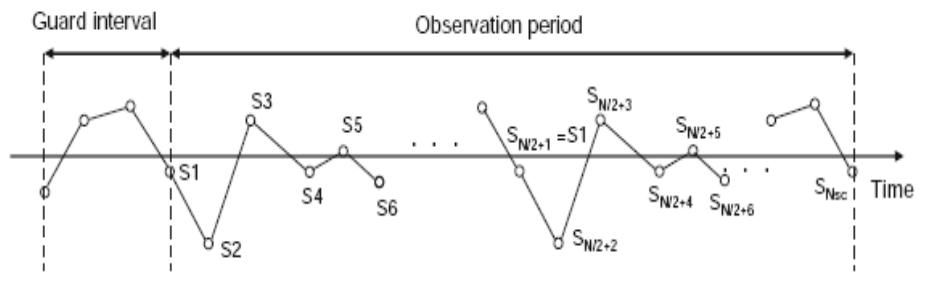

Fig. 3 the Schmidl synchronization format

Synchronization is one of the most critical functionalities for a CO-OFDM receiver. As discussed in the previous section, it can be divided into three levels of synchronization: DFT Window timing synchronization, carrier frequency offset synchronization, and subcarrier recovery. Figure 2 shows the time domain structure of an OFDM signal consisting of many OFDM symbols. Each OFDM symbol comprises a guard interval and an observation period. It is imperative that the start of the DFT window (i.e., the observation period) be determined properly because an improper DFT window will result in intersymbol interference (ISI) and intercarrier interference (ICI) [7].

One of the popular methods for window synchronization was proposed by Schmidl and Cox.[4]. In such a method, a pilot symbol or preamble is transmitted that consists of two identical segments, as shown in Figure 3, which can be expressed as:

$$
s_{m}=s_{m-} N_{\frac{s c}{2}}, m \in\left[\frac{N_{s c}}{2}+1, N_{s c}\right]
$$


Where $s_{m}$ is the $m$ th sample with a random value when $m$ is from 1 to $N_{s c} / 2$. Assuming a time-invariant channel impulse response function $\mathrm{h}(\mathrm{t})$ the sampled received signal has the following form:

$$
r_{m}=r\left(\frac{m t_{s}}{N_{s c}}\right)=r_{m}^{0} e^{j\left(\omega_{o f f} \frac{m t_{s}}{N_{s c}}\right)}+n_{m}
$$

We have assumed that the constant phase across the entire OFDM symbol, or $\Delta \varphi$ equals zero in Eq. (9). The delineation can be identified by studying the following correlation function [4] defined as :

$$
R(d)=\sum_{m=1}^{N_{s c} / 2} r_{m+d}^{*} r_{m+d+N_{s c} / 2}
$$

The principle is based on the fact that the second half of $r_{m}$ is identical to the first half except for a phase shift. Assuming the frequency offset $\omega_{\text {off }}$ is small to start with, we anticipate that when $d=0$, the correlation function $\mathrm{R}(\mathrm{d})$ reaches its maximum value. The correlation function can be normalized to its maximum value given by:

$$
M(d)=\left|\frac{R_{d}}{S_{d}}\right|
$$

And

$$
\mathrm{S}_{\mathrm{d}}=\sqrt{\left(\sum_{\mathrm{m}=1}^{\mathrm{N}_{\mathrm{sc}} / 2} \mathrm{r}_{\mathrm{m}+\mathrm{d}}^{2}\right)\left(\sum_{\mathrm{m}=1}^{\mathrm{N}_{\mathrm{sc}} / 2} \mathrm{r}^{2}{ }_{\left.\mathrm{m}+\mathrm{d}+\mathrm{N}_{\mathrm{sc}} / 2\right)}\right.}
$$

Where $M(d)$ is defined as the DFT window synchronization timing metric. The optimal timing metric has its peak at the correct starting point of the OFDM symbol that is:

$$
\mathrm{d}_{\mathrm{opt}}=\arg \{\max [\mathrm{M}(\mathrm{d})]\}
$$

Where $\arg \{\max [M(d)]\}$ in general stands for searching the optimal argument of $d$ that maximizes the objective function of $M(d)$, and $d_{o p t}$ stands for the optimal timing point.

\section{Simulation And Result}

We have conducted a matlab simulation for the coherent optical OFDM presented in figure 1 to confirm the DFT

\begin{tabular}{|c|c|}
\hline Data rate $(\mathrm{G} / \mathrm{s})$ & 10 \\
\hline Modulation & QAM \\
\hline $\mathrm{M}$ & 4 \\
\hline FFT Size & 128 \\
\hline $\begin{array}{l}\text { L(number of } \\
\text { samples of the } \\
\text { training symbol) }\end{array}$ & 256 \\
\hline $\begin{array}{c}\text { Number of } \\
\text { samples per bit }\end{array}$ & 4 \\
\hline Number of blocks & 1 \\
\hline $\begin{array}{l}\text { Number of } \\
\text { symbols }\end{array}$ & 100 \\
\hline Number of frames & 100 \\
\hline CP length & $1 / 8$ \\
\hline Wavelength (nm) & 1.55 \\
\hline $\begin{array}{l}\text { Dispersion } \\
(\mathrm{ps} / \mathrm{nm})\end{array}$ & 17000 \\
\hline $\begin{array}{l}\text { Laser linewidth } \\
(\mathrm{KHz})\end{array}$ & 100 \\
\hline
\end{tabular}
window synchronization using the Schmidl format for a $\mathrm{CO}$ OFDM system at $10 \mathrm{~Gb} / \mathrm{s}$ under the influence of chromatic dispersion, linewidth, and optical-to-signal noise ratio (OSNR).

TABLE I. SIMULATION PARAMETERS

The OFDM system parameters used for the simulation are described in the TABLE I mentioned above.

\section{A. Timing metric for $\mathrm{CO}-\mathrm{OFDM}$ systems}

This plot shows that the peak of the timing metric decreases from an ideal value of 1 to approximately 0.3 when the SNR is $0 \mathrm{~dB}$. All curves show the flat platform corresponding to the guard interval inserted in the beginning of each symbol. 


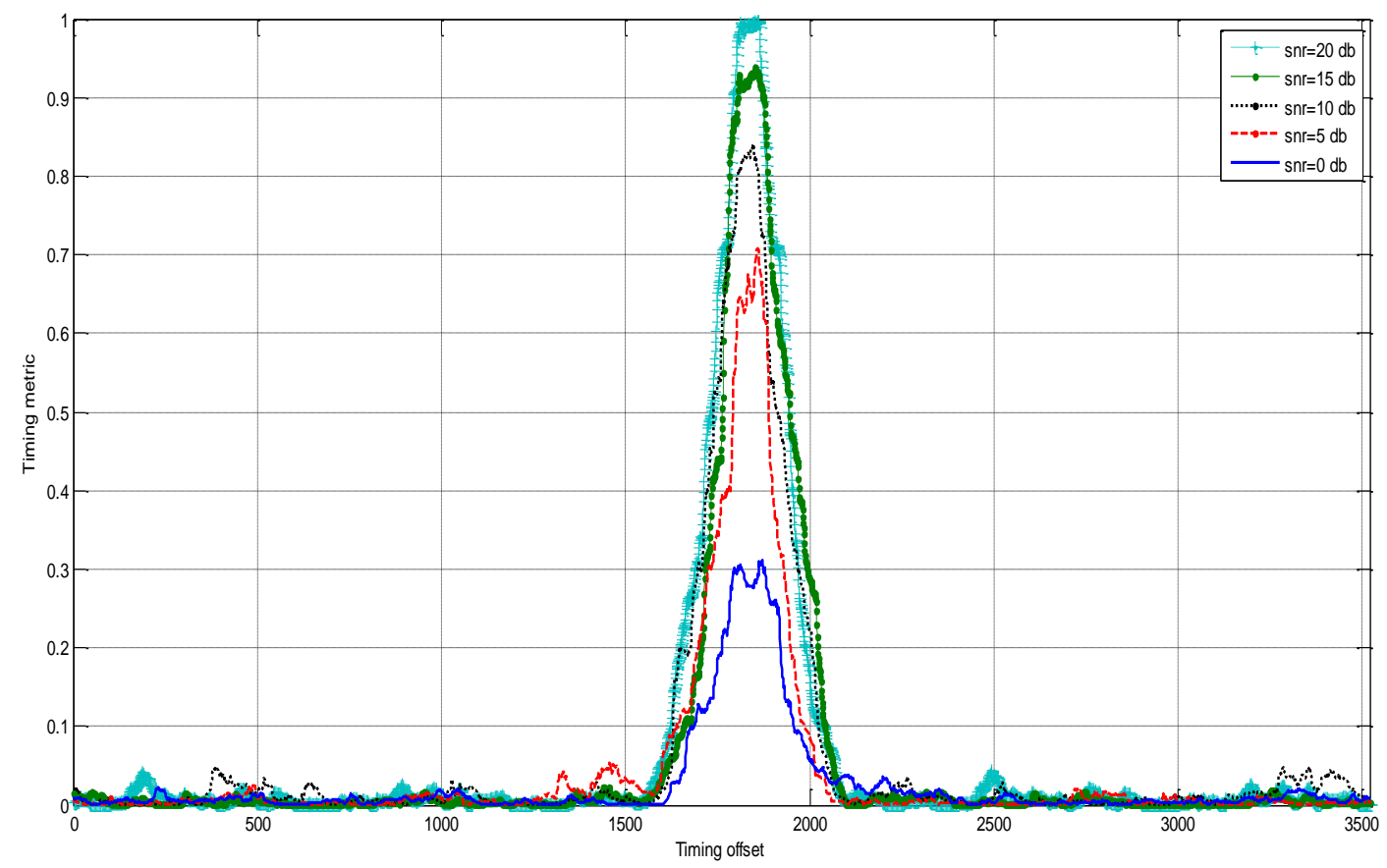

Fig. 4 Timing metric for CO-OFDM systems

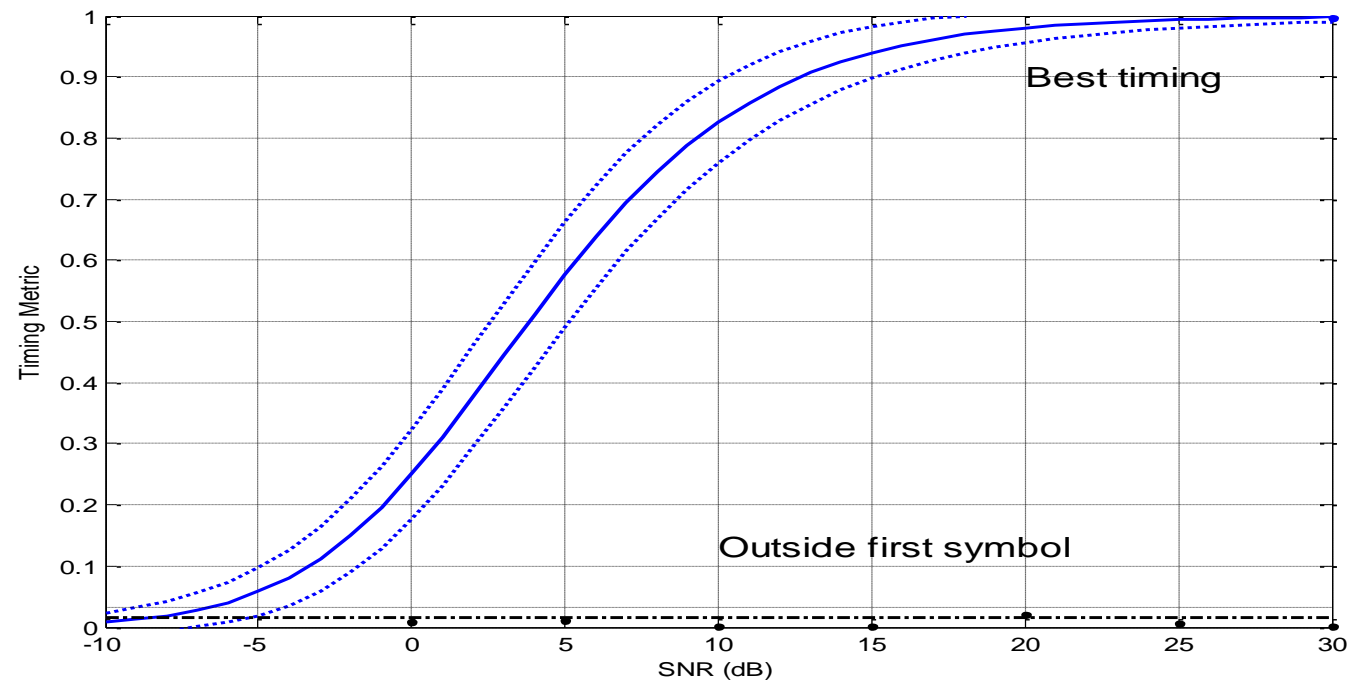

Fig. 5 Expected value of timing metric. (Dashed lines indicate three standard deviations)

This figure shows a plot of the expected value of timing metric $M(d)$ versus SNR at both the best timing instant and a point outside the training symbol. The dashed lines indicate three standard deviations from each curve.

We concluded that outside the training symbol the timing metric is null.

\section{B. Mean and Variance at correct timing}

The correct timing point was chosen at the start of the useful part of the first training symbol, the plots show that variance of timing metric is lower than the mean of timing metric. 

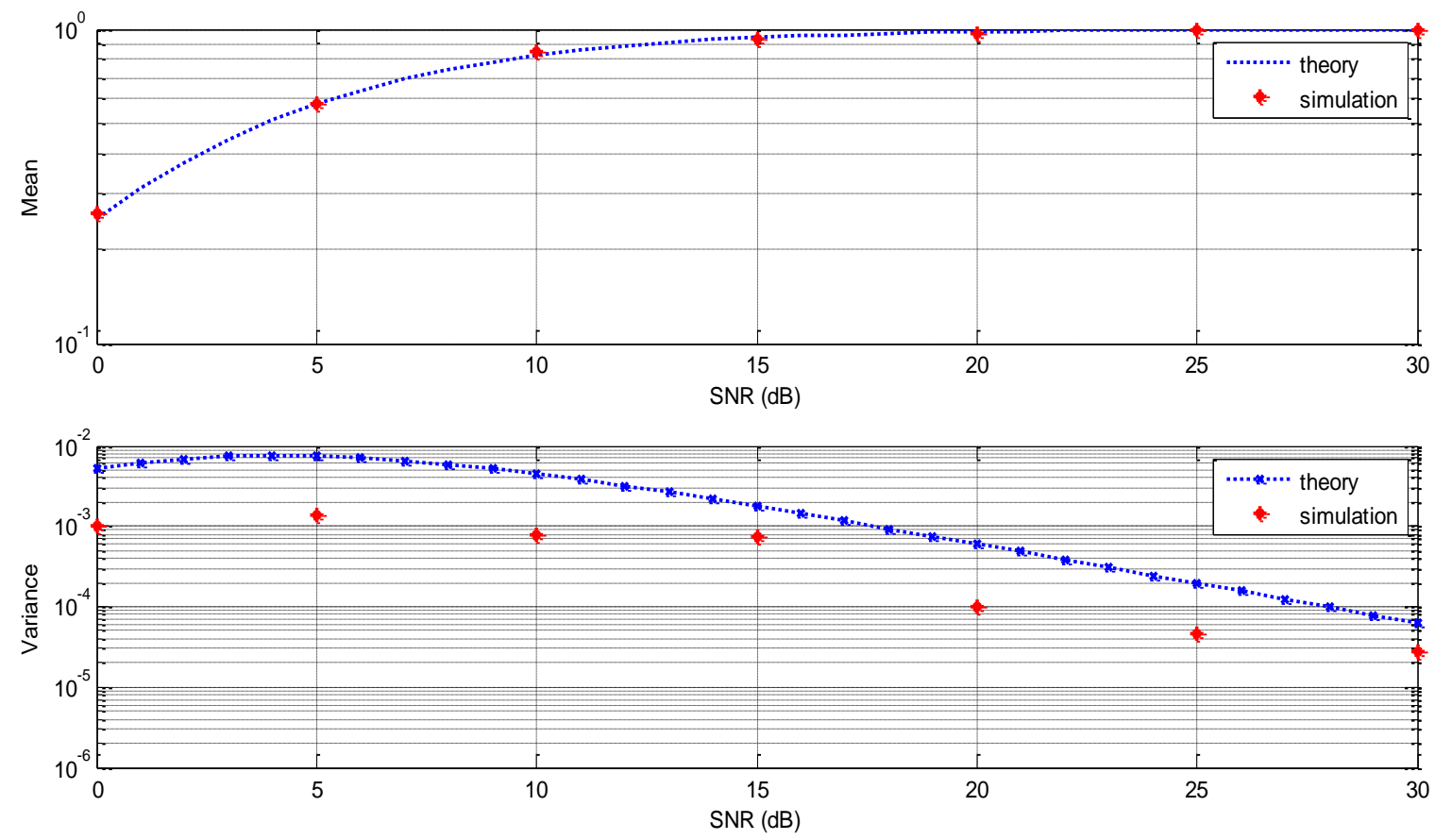

Fig. 6 Mean and Variance at correct timing
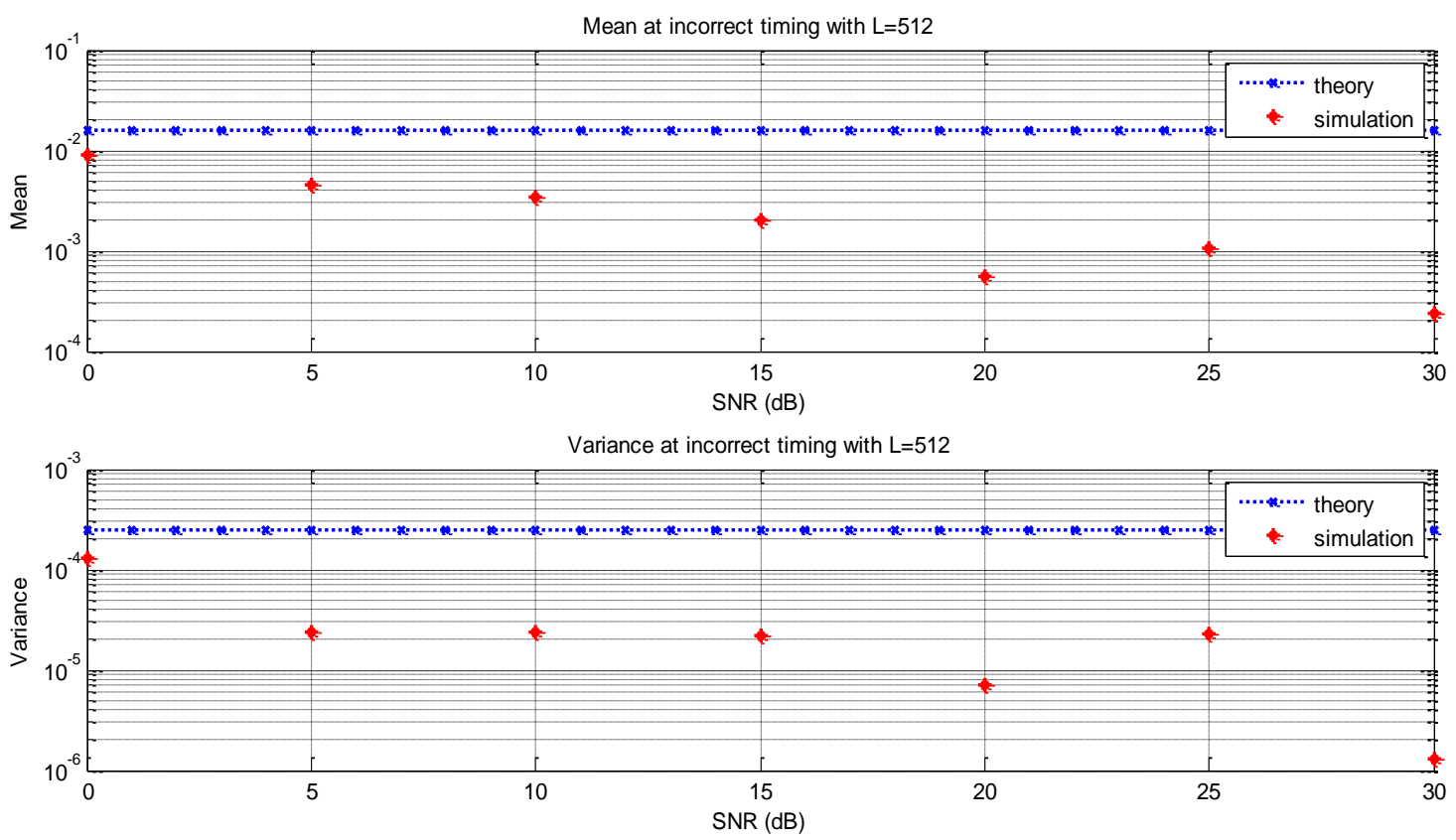

Fig. 7 Mean and variance at incorrect timing

Figure 6 shows the result of simulation for the correct timing.

\section{Mean and Variance at incorrect timing}

Figures 7 and 8 show the mean and variance of the incorrect timing. The incorrect timing point was chosen one symbol after the training symbol, the performance of the variance of timing metric is lower than the performance of the mean of the timing metric.
Some plot shows that there's a differentiation between the theory and simulation result and this lead on the two terms $r_{m+d}^{*}$ and $r_{m+d+N_{s c / 2}}$ which are dependent and we consider in the simulations that are independent.

D. Influence of chromatic dispersion

This plot show that the increase of the chromatic dispersion leads on an increase of the timing offset and the minimum timing offset correspond on a null chromatic dispersion. 


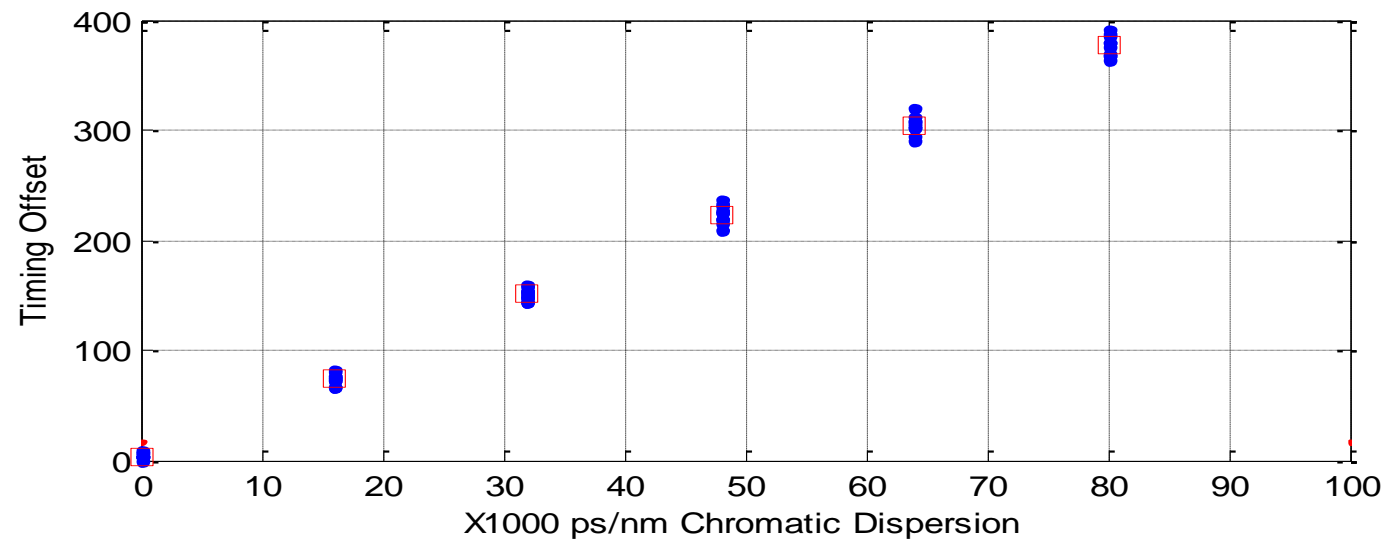

Fig. 8 Influence of chromatic dispersion

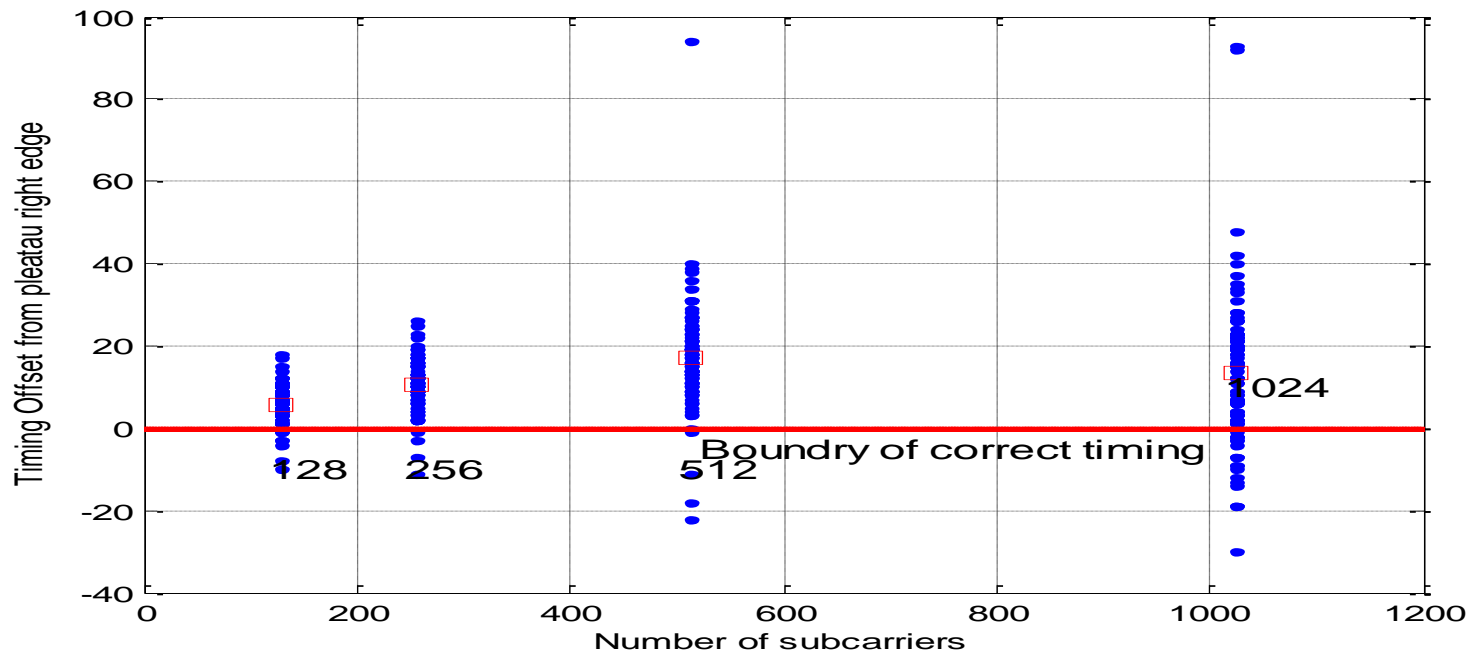

Fig. 9 Influence of number of subcarriers

\section{E. Influence of number of subcarriers}

This figure shows that the increase of the number of subcarrier lead on an increase of the timing offset; so to avoid this timing offset we must insert in the OFDM symbol in the beginning and in the end a sufficient number of zeros to avoid this increase of timing offset.

\section{CONCLUSION}

In this paper, we evaluated the performance of shmidl and cox algorithm of synchronization in coherent optical OFDM system. This method present a flat plateau in the timing offset versus SNR, so we aim in future works to eliminate this plateau and improve the performance of estimators in terms of mean and variance of timing metric and the complexity of synchronization algorithm.

\section{REFERENCES}

[1] Shieh.W and Authadage C. coherent optical orthogonal frequency division multiplexing; electronic letters.42(10),587-589 (2006).
[2] Shieh.W,Yang.Q, and MA.Y.107 Gb/s coherent optical ofdm transmission over $1000 \mathrm{~km}$ SSMF fiber using orthogonal band multiplexing, optics express,16(9),6378-6386 (2008).

[3] Ma CP, Kuo JW. Orthogonal frequency division multiplex with multilevel technology in optical storage application. Jpn J Appl Physics Part 1: Regular Papers Short Notes Rev Papers 2004;43:4876-8.

[4] Schmidl TM, Cox DC. Robust frequency and timing synchronization for OFDM. IEEE Trans Commun 1997;45:1613-21.

[5] Minn H, Bhargava VK, Letaief KB. A robust timing and frequency synchronization for OFDM systems. IEEE Trans Wireless Commun 2003;2:822-39.

[6] Hara S, Prasad R. Multicarrier Techniques for 4G Mobile Communications. Boston: Artech House; 2003.

[7] Hanzo L, Munster M, Choi BJ, Keller T. OFDM and MC-CDMA for Broadband Multi-User Communications, WLANs and Broadcasting. New York: Wiley; 2003.

[8] Bauml RW, Fischer RFH, Huber JB. Reducing the peak-to-average power ratio of multicarrier modulation by selected mapping. IET Electron Lett 1996;32:2056-7.

[9] Friese M. OFDM signals with low crest-factor. In: Proc. 1997 IEEE Global Telecommun. Conf; 1997. pp. 290-4. 